Preface

\title{
Preface: Altered Fibrinolysis-Clinical Impact and Diagnostic Challenges
}

\author{
Julie Brogaard Larsen, MD, $\mathrm{PhD}^{1}$ Ton Lisman, $\mathrm{PhD}^{3}$ \\ 1 Thrombosis and Hemostasis Research Unit, Department of Clinical \\ Biochemistry, Aarhus University Hospital, Aarhus, Denmark \\ ${ }^{2}$ Department of Clinical Medicine, Aarhus University, Aarhus, \\ Denmark \\ ${ }^{3}$ Department of Surgery, Surgical Research Laboratory and Section of \\ Hepatobiliary Surgery and Liver Transplantation, University of \\ Groningen, University Medical Center Groningen, Groningen, \\ The Netherlands
}

Semin Thromb Hemost 2021;47:477-479.

Fibrinolysis is the physiological and continuous process of plasmin-mediated fibrin breakdown, which is regulated to keep the balance between ensuring continued blood supply to end organs and maintaining hemostasis in the face of vascular damage. ${ }^{1}$ The importance of a well-regulated fibrinolytic system is illustrated by rare inherited and acquired disorders of fibrinolysis with severe bleeding phenotypes. $^{2}$ Moreover, a growing body of evidence demonstrates that altered fibrinolysis plays a pivotal role in the pathophysiology of a range of clinical conditions, and markers of fibrinolysis hold the potential to predict adverse events such as bleeding and thrombosis. ${ }^{3}$ Finally, drugs which modulate fibrinolysis have lifesaving potential and are used to treat millions of patients each year worldwide, both in the form of profibrinolytics used for thrombolysis, for example, in ischemic stroke and pulmonary emboli, and as antifibrinolytics used to mitigate traumatic, surgical, and postpartum bleeding.

In the current issue of Seminars in Thrombosis and Hemostasis, we have assembled review articles dealing with the fibrinolytic system in pathophysiology, diagnosis, and treatment of clinical disorders, including arterial and venous thrombosis, cancer, sepsis, and liver disease to traumatic brain injury. Furthermore, the use of tranexamic acid in trauma and pediatric surgery is reviewed, and the implication of an emerging treatment, ischemic preconditioning, for fibrinolysis in ischemic stroke is discussed.

Address for correspondence Anne-Mette Hvas, MD, PhD, Thrombosis and Hemostasis Research Unit, Department of Clinical Biochemistry, Aarhus University Hospital, Palle JuulJensens Boulevard 99, DK - 8200

Aarhus N, Denmark

(e-mail: am.hvas@dadlnet.dk).

\section{Hypofibrinolysis and Thromboembolic Risk}

Fibrinolysis is of key importance in maintaining vessel patency, or regaining it in case of obstruction by a thrombus. Impaired fibrinolysis may contribute to both first and recurrent venous thromboembolism (VTE). The role of impaired fibrinolysis in VTE and risk of recurrence is therefore reviewed by Undas. ${ }^{4}$ The author summarizes results from studies performed by the author and other groups, which indicate that markers of fibrinolysis may be useful in the acute phase as prognosticators of survival and of both safety and efficacy of thrombolysis treatment. Fibrinolysis may also have relevance in VTE in the long term, as fibrinolysis appears impaired in VTE patients, not only immediately after VTE but also in the long term, and fibrin clot lysis time may aid in predicting recurrence risk.

Not only venous but also arterial thrombosis risk may be influenced by impaired fibrinolysis. The comprehensive review by Kietsiriroje et al summarizes current evidence for altered fibrinolysis in patients with arterial thrombosis including stable and acute coronary disease, ischemic stroke, and peripheral artery disease. ${ }^{5}$ These diseases have long been considered to be mediated mainly by increased platelet activation and have consequently been treated with antiplatelet agents. However, the authors emphasize that impaired fibrinolysis is prevalent in these patients. This constitutes a residual risk factor for adverse vascular outcomes in these patients with arterial disease which is not treated sufficiently with antiplatelet therapy alone. Thus, (c) 2021. Thieme. All rights reserved. Thieme Medical Publishers, Inc., 333 Seventh Avenue, 18th Floor, New York, NY 10001, USA
DOI https://doi.org/ 10.1055/s-0041-1725100. ISSN 0094-6176.
Clinical Impact and Diagnostic Challenges; Guest Editors: AnneMette Hvas, MD, PhD, Julie Brogaard Larsen, MD, PhD, and Ton Lisman, MSc, PhD 
targeting proteins in the fibrinolytic system represents a possible strategy to improve outcome in this population.

Antiphospholipid syndrome (APS) represents a special prothrombotic condition with high risk of both arterial and venous thrombosis. ${ }^{6}$ The pathophysiological mechanisms by which antiphospholipid antibodies cause thrombosis have not been fully elucidated. The review by Antovic and Bruzelius provides an overview of the current literature on fibrinolysis in APS patients, ${ }^{7}$ showing that fibrinolysis may be impaired through multiple different mechanisms in APS and that the understanding of fibrinolysis as a key player in APS has implications both for the diagnosis and treatment of APSrelated thrombosis.

\section{Hyperfibrinolysis: Bleeding and Antifibrinolytic Agents}

In contrast to impaired fibrinolysis, which may contribute to both venous and arterial thrombosis, hyperfibrinolysis may be a major contributor to bleeding-related mortality. Classical conditions associated with hyperfibrinolysis-associated bleeding are surgery and trauma. Current knowledge on fibrinolytic alterations during surgery is reviewed by Colomina and colleagues. ${ }^{8}$ Profound and complex hemostatic changes appear during surgery, including activation of the endothelium and hemostasis, hemodilution, and bleedingrelated loss of both pro- and antifibrinolytic factors. In patients with unexpected surgical bleeding, quick assessment of fibrinolysis and subsequent correction with antifibrinolytic agents (e.g., tranexamic acid [TXA]) may be lifesaving, as the authors demonstrate.

The concept of trauma-induced coagulopathy has been studied to a great extent and hyperfibrinolysis is well recognized. Therefore, in their review, Roberts and colleagues ask the question: Why do we not treat more trauma patients with TXA $?^{9}$ In their review, they summarize the compelling evidence for the life-saving potential of early TXA treatment in both major and minor trauma and advocate for more widespread use of TXA on a global scale. However, one subgroup of trauma patients where the fibrinolytic system and the use of TXA have only been sparsely investigated is isolated traumatic brain injury. The review by Anderson and colleagues summarizes current knowledge on fibrinolytic disturbances in isolated traumatic brain injury and points to future directions for improving diagnosis and management of fibrinolytic disturbances in this patient group. ${ }^{10}$

The use of TXA is still not fully established in pediatric surgery, and especially dosing regimens have been subjects of uncertainty. Hovgesen and colleagues review the literature on the use of antifibrinolytics in trauma or surgery in children. ${ }^{11}$ Regarding trauma, not much evidence exists; however, the authors include 50 randomized studies on TXA or other antifibrinolytics in pediatric surgery in their systematic review and demonstrate that the use of antifibrinolytics is effective in reducing bleeding in both cardiac and noncardiac surgery. Moreover, TXA was shown to be safe in children and can be used following a weight-based dosing regimen comparable to that recommended for adults.
Cancer patients are at high risk of thrombosis, and at the same time, these patients are likely to suffer from bleeding complications caused by both the disease and its treatment, and both thrombosis and bleeding contribute significantly to morbidity and mortality in this patient group. ${ }^{12,13}$ Hyperfibrinolysis is a well-known complication of acute promyelocytic leukemia, but the contribution of altered fibrinolysis to bleeding and thrombosis risk in other cancer types has not received as much attention. Two reviews by Bønløkke and colleagues and Winther-Larsen and colleagues focus on fibrinolysis in patients with hematological cancers and in patients with solid tumors, respectively. ${ }^{14,15}$ Regarding hematological cancers, only few studies have investigated fibrinolysis and most of these studies were performed quite a long time ago. The authors conclude that multiple myeloma and myeloproliferative disease appear to be associated with impaired fibrinolysis, while no clear evidence exists for enhanced or impaired fibrinolysis in lymphoblastic leukemias. ${ }^{14}$ In patients with solid tumors, only a few case reports are published on cases of primary hyperfibrinolysis, mainly in prostate and pancreatic cancer. ${ }^{15}$ Thus, the subject area of fibrinolysis in cancer holds a strong potential for future research.

\section{Fibrinolysis in Sepsis or Liver Disease}

In some clinical conditions, both pro- and antifibrinolytic changes may occur, and especially in the critically ill patient, it may not be easily predictable which way the balance tips. Laboratory assessment of fibrinolysis is currently challenged by the lack of standardized biomarkers available in the routine clinical setting. New biomarkers of fibrinolysis may hold potential to characterize the individual patients' fibrinolytic capacity and thus assess bleeding and/or thrombosis risk and guide hemostatic support as well as anticoagulant therapy. The role of fibrinolysis biomarkers in sepsis is reviewed by Larsen and Hvas. ${ }^{16}$ It has long been a paradigm that sepsis patients have impaired fibrinolysis, and this may be true for a majority of sepsis patients. For some patients, however, fibrin formation is severely impaired or the rate of fibrinolysis could be normal or even enhanced. The review summarizes existing knowledge on both functional assays and circulating fibrinolysis markers in sepsis. The authors conclude that plasminogen activator inhibitor type 1 , plasma-based turbidimetric fibrin clot lysis assays, and modified viscoelastic tests hold the greatest future potential for fibrinolytic assessment in this patient group. On a related note, von Meijenfeldt and Lisman review our current knowledge on fibrinolysis in another patient group which suffers from profound hemostatic changes, namely, patients with liver dysfunction. ${ }^{17}$ This patient group has historically been considered to suffer from hyperfibrinolysis with subsequent bleeding risk; however, the authors summarize more recent evidence which questions this paradigm and reveals that patients with liver disease are a heterogeneous group which may suffer from both enhanced and impaired fibrinolysis depending on the type, severity, and acuteness of their liver disease. New biomarkers of fibrin formation and lysis 
capacity could be of great value to assess bleeding and thrombosis risk in this patient group.

\section{Ischemic Conditioning: An Emerging Intervention with Multiple Effects}

Finally, ischemic conditioning is emerging as a novel intervention with possible influence on fibrinolysis. Initially introduced to protect against ischemia-reperfusion injury and thus alleviate tissue damage after acute coronary syndrome, this therapy may also exert other beneficial effects. In their review, Krag and Blauenfeldt summarize emerging evidence that ischemic conditioning may stimulate endogenous fibrinolysis and thus could present a novel, noninvasive strategy in the treatment of other thrombotic conditions such as ischemic stroke. ${ }^{18}$

In summary, this issue of Seminars in Thrombosis and Hemostasis highlights several clinical conditions complicated by altered fibrinolysis and treatment hereof. Besides, current diagnostic approaches are discussed. The reviews also stress the research needed to further improve current knowledge within the subject area of fibrinolysis in different clinical entities. We hope that readers enjoy the content and that it leads to further discussion of the variety of topics presented herein.

Conflict of Interest

A-M.H. reports grants from CSL Behring and other from CSL Behring, Bayer, Boehinger-Ingelheim, and Astellas, outside the submitted work.

\section{References}

1 Longstaff C, Kolev K. Basic mechanisms and regulation of fibrinolysis. J Thromb Haemost 2015;13(Suppl 1):S98-S105

2 Kolev K, Longstaff C. Bleeding related to disturbed fibrinolysis. Br J Haematol 2016;175(01):12-23
3 Lisman T. Decreased plasma fibrinolytic potential as a risk for venous and arterial thrombosis. Semin Thromb Hemost 2017;43 (02):178-184

4 Undas A. Fibrinolysis in venous thromboembolism. Semin Thromb Hemost 2021;47(05):480-489

5 Kietsiriroje N, Ariëns RAS, Ajjan RA. Fibrinolysis in acute and chronic cardiovascular disease. Semin Thromb Hemost 2021;47 (05):490-505

6 Ruiz-Irastorza G, Crowther M, Branch W, Khamashta MA. Antiphospholipid syndrome. Lancet 2010;376(9751):1498-1509

7 Antovic A, Bruzelius M. Impaired fibrinolysis in the antiphospholipid syndrome. Semin Thromb Hemost 2021;47(05):506-511

8 Colomina MJ, Mendéz E, Sabate A. Altered fibrinolysis during and after surgery. Semin Thromb Hemost 2021;47(05):512-519

9 Roberts I, Brenner A, Shakur-Still H. Tranexamic acid treatment for trauma victims. Semin Thromb Hemost 2021;47(05):520-526

10 Anderson TN, Rowell SE, Farrell DH. Fibrinolysis in traumatic brain injury: diagnosis, management, and clinical considerations. Semin Thromb Hemost 2021;47(05):527-537

11 Hovgesen NT, Larsen JB, Fenger-Eriksen C, Hansen AK, Hvas AM. Efficacy and safety of antifibrinolytic drugs in pediatric surgery: a systematic review. Semin Thromb Hemost 2021;47(05):538-568

12 Khorana AA, Francis CW, Culakova E, Kuderer NM, Lyman GH. Thromboembolism is a leading cause of death in cancer patients receiving outpatient chemotherapy. J Thromb Haemost 2007;5 (03):632-634

13 Weycker D, Hatfield M, Grossman A, et al. Risk and consequences of chemotherapy-induced thrombocytopenia in US clinical practice. BMC Cancer 2019;19(01):151

14 Bønløkke ST, Ommen HB, Hvas AM. Altered fibrinolysis in hematological malignances. Semin Thromb Hemost 2021;47(05):569-580

15 Winther-Larsen A, Sandfeld-Paulsen B, Hvas AM. Hyperfibrinolysis in patients with solid malignant neoplasms: a systematic review. Semin Thromb Hemost 2021;47(05):581-588

16 Larsen JB, Hvas AM. Fibrinolytic alterations in sepsis: biomarkers and future treatment targets. Semin Thromb Hemost 2021;47 (05):589-600

17 Von Meijenfeldt FA, Lisman T. Fibrinolysis in patients with liver disease. Semin Thromb Hemost 2021;47(05):601-609

18 Krag AE, Blauenfeldt RA. Fibrinolysis and remote ischemic conditioning: mechanisms and treatment perspectives in stroke. Semin Thromb Hemost 2021;47(05):610-620 